\title{
Normalized Power Variance: A New Field Orthogonal to Power in EEG Analysis
}

Yasunori Aoki ( $\nabla$ mi_kotoginn@yahoo.co.jp )

Osaka University Graduate School of Medicine

Hiroaki Kazui

University of Kochi

Roberto D. Pascual-Marqui

University Hospital of Psychiatry

Ricardo Bruña

La Laguna University

Kenji Yoshiyama

Osaka University Graduate School of Medicine

Tamiki Wada

Osaka University Graduate School of Medicine

Hideki Kanemoto

Osaka University Graduate School of Medicine

Yukiko Suzuki

Osaka University Graduate School of Medicine

Takashi Suehiro

Osaka University Graduate School of Medicine

Yuto Satake

Osaka University Graduate School of Medicine

Maki Yamakawa

Osaka University Graduate School of Medicine

Masahiro Hata

Osaka University Graduate School of Medicine

Leonides Canuet

Nuestra Senora del Rosario hospital

Ryouhei Ishii

Osaka Prefecture University

Masao Iwase

Osaka University Graduate School of Medicine

Manabu Ikeda

Osaka University Graduate School of Medicine 


\section{Research Article}

Keywords: Normalized Power Variance (NPV), Electroencephalography (EEG), Information geometry, Gamma distribution, dimension, Idiopathic normal pressure hydrocephalus (iNPH)

Posted Date: July 22nd, 2021

DOI: https://doi.org/10.21203/rs.3.rs-736229/v1

License: (c) (i) This work is licensed under a Creative Commons Attribution 4.0 International License. Read Full License

Version of Record: A version of this preprint was published at Clinical EEG and Neuroscience on March 29th, 2022. See the published version at https://doi.org/10.1177/15500594221088736. 


\section{Normalized Power Variance: a new field orthogonal to Power}

\section{2 in EEG analysis}

3 Yasunori Aoki $^{\text {a,b, }}$, Hiroaki Kazui ${ }^{\mathrm{c}}$, Roberto D. Pascual-Marqui ${ }^{\mathrm{d}}$, Ricardo Bruña, ${ }^{\mathrm{e}, \mathrm{f}}$, Kenji

4 Yoshiyama $^{\text {a }}$, Tamiki Wada ${ }^{\text {a }}$, Hideki Kanemoto ${ }^{\text {a }}$, Yukiko Suzuki ${ }^{\text {a }}$, Takashi Suehiro ${ }^{\text {a }}$, Yuto

5 Satake $^{\mathrm{a}}$, Maki Yamakawa ${ }^{\mathrm{a}}$, Masahiro Hata ${ }^{\mathrm{a}}$, Leonides Canuet ${ }^{\mathrm{g}}$, Ryouhei Ishii ${ }^{\mathrm{a}, \mathrm{h}}$, Masao Iwase $^{\mathrm{a}} \&$ Manabu Ikeda ${ }^{\mathrm{a}}$

7

$8{ }^{a}$ Department of Psychiatry, Graduate School of Medicine, Osaka University, Suita,

9 Osaka, Japan

10 bepartment of Psychiatry, Nippon Life Hospital, Osaka, Japan

$11{ }^{\mathrm{c}}$ Department of Neuropsychiatry, Kochi Medical School, Kochi University

$12{ }^{\mathrm{d}}$ The KEY Institute for Brain-Mind Research, University Hospital of Psychiatry, Zurich,

13 Switzerland

14 e UCM-UPM Centre for Biomedical Technology, Laboratory of Cognitive and

15 Computational Neuroscience, Complutense University of Madrid, Madrid, Spain

$16{ }^{\mathrm{f}}$ Department of Electrical Engineering, La Laguna University, Tenerife, Spain

$17{ }^{\mathrm{g}}$ Neurology department, Nuestra Senora del Rosario hospital, Madrid, Spain

18 hraduate School of Comprehensive Rehabilitation, Osaka Prefecture University, Osaka,

19 Japan

21 Corresponding author

22 Yasunori Aoki, M.D., Ph, D.

23 Department of Psychiatry, Osaka University Graduate School of Medicine

24 D3 2-2 Yamada-oka, Suita, Osaka 565-0871, Japan

25 Phone: $+81-6-6879-3051$

26 Email: mi_kotoginn@yahoo.co.jp 


\section{Abstract}

30 To date, electroencephalogram (EEG) has been used in the diagnosis of epilepsy, dementia, and disturbance of consciousness via the inspection of EEG waves. In addition, EEG power analysis combined with a source estimation method like exactlow-resolution-brain-electromagnetic-tomography (eLORETA), which calculates the power of cortical electrical activity from EEG data, has been widely used to investigate cortical electrical activity in both healthy individuals and neuropsychiatric patients. However, the recently developed field of mathematics "information geometry" indicates that EEG has another dimension orthogonal to power dimension - that of normalized power variance (NPV). By also introducing the idea of information geometry, a significantly faster convergent estimator of NPV was obtained. In this study, we applied this NPV analysis of eLORETA to idiopathic normal pressure hydrocephalus (iNPH) patients prior to a cerebrospinal fluid (CSF) shunt operation, where traditional power analysis could not detect any difference associated with CSF shunt operation outcome. NPV analysis detected significantly higher NPV values at the high convexity area in the beta frequency band between 17 shunt responders and 19 non-responders. Our findings demonstrated that EEG has another dimension - that of NPV, which contains a great deal of information about cortical electrical activity that can be useful in clinical practice.

Keywords: Normalized Power Variance (NPV), Electroencephalography (EEG), 50 Information geometry, Gamma distribution, dimension, Idiopathic normal pressure hydrocephalus (iNPH) 


\section{Introduction}

Electroencephalogram (EEG) noninvasively measures brain cortical electrical activity at a high sampling rate $(500-1000 \mathrm{~Hz})$ via electrodes placed on the scalp. In the cerebral cortex, neurons connect with other surrounding neurons via synapses and collectively give rise to electrical activity, which can be detected as EEG waves by these electrodes. Hans Berger, a German psychiatrist, first recorded human EEG in 1924 and dedicated many years to his studies before proving that EEG stems from brain electrical activity, rather than artifacts ${ }^{1}$. Finally, EEG gained widespread recognition and visual inspection of EEG waves has been used to support diagnoses of epilepsy, dementia, and disturbance of consciousness ever since. Owing to its high temporal resolution (1-2 milliseconds), EEG contains a large amount of information about cortical electrical activities. However, most of this information cannot be identified by visual inspection and, therefore, it is necessary to use various analysis methods (as outlined below) to extract it. Electrical activities are generated from each cerebral cortex and their linear mixture is captured by the electrodes on the scalp. So, it is necessary to estimate cerebral electrical activity from the EEG waves by solving a linear inverse problem. More specifically, discrete Fourier transform is applied, such that the EEG data recorded on electrodes are transformed into electrode position $\times$ frequency data. The linear inverse problem is then solved by multiplying this data by a spatial filter matrix, thereby achieving conversion of the electrode position $\times$ frequency data into cerebral cortex position $\times$ frequency data (source estimation method) ) $^{2,3}$. Exact low-resolution brain electromagnetic tomography (eLORETA) is a source estimation method that can reconstruct cortical electrical activity from EEG data with correct localization ${ }^{2,4-6}$. Therefore, power analysis with eLORETA has been widely used, providing valuable results in neuroimaging studies ${ }^{4,7-8}$.

Approaching EEG analysis from a different mathematical perspective, the cortical electrical activity approximately follows a specific type of probability distribution (namely, gamma distribution) ${ }^{9}$, so EEG analysis can be considered from the point of view of 'information geometry'. This recently developed mathematical field allows a probability distribution to be assigned to a Riemannian manifold equipped with a metric tensor, facilitating the investigation of probability distributions by means of geometry ${ }^{10}$. Information geometry indicates that cortical electrical activity has two orthogonal dimensions, namely, normalized power variance (NPV) and Power - and we can calculate a metric tensor and distance between probability distributions in these dimensions (NPV and Power) on the Riemannian manifold ${ }^{11}$. In addition, by applying a maximum likelihood estimation technique, a closed-form estimator of NPV was 
obtained, which converges to the true value significantly faster than is the case with a classical moment estimator ${ }^{12}$.

From the point of view of thermal and statistical physics, NPV sensitively reflects the instability of cortical electrical activity and can be an early warning signal of phase transition of the cortical electrical activity from the healthy state to the disease state ${ }^{13}$. In previous studies, we applied the moment estimator of NPV to EEG data in epilepsy and showed the high sensitivity of NPV with regard to the instability of cortical electrical activity at the epileptogenic electrode in the pre-seizure period and its stabilization after phase transition to the seizure period ${ }^{14}$. We also applied the moment estimator of NPV to eLORETA source estimated data in patients of idiopathic normal pressure hydrocephalus (iNPH) prior to a cerebrospinal fluid (CSF) shunt operation. Our results indicated that there was a significant difference between shunt responders and nonresponders appearing in NPV at the high-convexity area in the beta frequency band, but not in Power of eLORETA ${ }^{15}$.

In the present study, using information geometry, we demonstrated that the cortical electrical activity has another dimension - that of NPV - in addition to the Power dimension (as outlined in Methods). Traditional neuroimaging techniques such as fMRI, SPECT, and EEG power analysis only measured information in the Power dimension. We therefore explored whole dimensions (NPV and Power) of eLORETA cortical electrical activity using the closed-form estimator in iNPH patients prior to a CSF shunt operation and aimed to find presurgical differences in cortical electrical activity that associated with the CSF shunt operation outcome - a feat that traditional neuroimaging techniques have failed to achieve $\mathrm{e}^{15,16}$. Our findings indicate that NPV analysis represents a new field in EEG analysis, providing a great deal of useful information to be used in clinical practice and facilitating greater understanding of the mechanisms of neuropsychiatric diseases.

\section{Results}

\section{Demographic and clinical results}

Based on the CSF shunt operation outcome, 36 right-handed iNPH patients were classified into 17 shunt responders and 19 non-responders (as outlined in Methods). Almost all of the shunt responder patients had improvement in the gait function only, while one patient had both gait and cognitive improvement and two patients had cognitive improvement only. Table 1 displays demographic and clinical characteristics of the subjects, showing that there was no significant difference between shunt responders and non-responders regarding gender, age, and initial clinical scores. The 
ratio of shunt responders to non-responders was relatively low, as our classification criteria for the CSF shunt operation outcome were stricter than those of the Japanese Clinical Guidelines, in order to classify patients that had shown significant improvements in gait or cognition as shunt responders ${ }^{17}$.

\section{eLORETA-NPV analysis results}

There was a significant difference in location along the NPV axis on the Riemannian manifold between shunt responders and non-responders in eLORETA cortical electrical activities measured by G-test with the symmetrized Bregman Divergence. Specifically, shunt responders had significantly higher NPV values of cortical electrical activity compared to non-responders at the high-convexity area (i.e., cingulate gyrus, paracentral lobule, and medial frontal gyrus) in the beta frequency band, as shown in Figs. 1, 2, 3 [extreme $p=0.016$ at the paracentral lobule $(X=5, Y=-15, Z$ $=50(\mathrm{~mm}) ;$ MNI coordinates $)]$.

\section{eLORETA power analysis results}

There was no significant difference in location along the power axis on the Riemannian manifold between shunt responders and non-responders. In addition, there was no significant difference between shunt responders and non-responders with regard to the location in the whole space on the Riemannian manifold.

\section{Discussion}

Information geometry demonstrated that the cortical electrical activity has another dimension in addition to the Power dimension on the Riemannian manifold - that of NPV (as outlined in Methods). Given that this point had so far received little attention, in the present study we investigated whole dimensions (NPV and Power) of cortical electrical activity using eLORETA with the closed-form estimator of NPV in iNPH patients prior to them having a CSF shunt operation. Our main finding was that a significant difference in location between shunt responders and non-responders resided only along the NPV dimension - but not along the Power dimension- on the Riemannian manifold.

In a previous study, using eLORETA analysis with the classical moment estimator of NPV, we found that shunt responders had significantly higher NPV values at the high-convexity area in the beta frequency band compared to non-responders (extreme $p$ at $\mathrm{X}=5, \mathrm{Y}=-15, \mathrm{Z}=45(\mathrm{~mm}) ; \mathrm{MNI}$ coordinates $)^{15}$. In the present study, eLORETA 
analysis with the closed-form estimator of NPV demonstrated almost the same spatial configuration of NPV in shunt responders and non-responders (Figs. 1,2) and detected a significant difference in NPV between shunt responders and non-responders at almost the same cortical point (extreme $p$ at $\mathrm{X}=5, \mathrm{Y}=-15, \mathrm{Z}=50(\mathrm{~mm})$; MNI coordinates) (Fig. 3). In a previous study, we used the classical moment estimator of NPV and employed a moving average filter method to compensate for the slow convergence of the moment estimator. In the present study, the fact that the eLORETA analysis with the closed-form estimator of NPV obtained practically the same results without using the moving average filter method suggests that the closed-form estimator of NPV has significantly faster convergence than the moment estimator previously demonstrated by Zhi-Sheng and $\mathrm{Nan}^{12}$.

From the perspective of thermal and statistical physics, NPV sensitively reflects the instability of cortical electrical activity and detects early changes in cortical electrical activity prior to phase transition from the healthy state to the disease state ${ }^{13}$. Musha et al who is renowned for his 1/f fluctuation research, reported that NPV analysis of EEG data could identify pre-Alzheimer's disease patients (who later developed Alzheimer's disease in $12-18$ months) from healthy subjects with a low false positive rate of $15 \%{ }^{18}$. We also applied NPV analysis to EEG data in epilepsy and showed sensitive detectability of NPV, visualizing the early surge of NPV value at the epileptogenic electrode prior to seizure onset ${ }^{14}$. In addition, we applied eLORETA-NPV with the moment estimator to EEG data of iNPH patients prior to them having CSF shunt operation and found a significant difference in NPV between shunt responders and nonresponders at the high-convexity area ${ }^{15}$. The high-convexity area is compressed by dilated ventricles with accumulated CSF is an area that is attracting attention due to its association with responsiveness to CSF shunt operation ${ }^{19-21}$. These past results, taken together with our present findings, suggest that NPV has the capacity for the early detection of cortical impairment prior to phase transition from the healthy state to the disease state or from reversible state to irreversible state for treatment.

From the perspective of information geometry, the cortical electrical activity has two orthogonal dimensions - NPV and Power- on the Riemannian manifold, as outlined in Methods. Traditional EEG power analysis methods have only measured power dimension information of the cortical electrical activity. Other neuroimaging methods such as fMRI and SPECT measure secondary hemodynamic and metabolic changes to the neuronal activity with high spatial resolution, albeit at low temporal resolution, and thus contain almost exclusively power dimension information of the neuronal activity. In this study, using the symmetrized Bregman Divergence, we 
observed a significant distance between shunt responders and non-responders along the NPV dimension — but not along the Power dimension — on the Riemannian manifold, which indicates the importance of exploring whole dimensions of cortical electrical activity.

Brain aging occurs due to the accumulation of age-related physiological changes in the brain neuronal population. In a previous study, using NPV analysis, we investigated age-related changes in healthy individuals and found that the levels of NPV of cortical electrical activity increased from the young to middle-aged group and decreased from the middle-aged to the elderly group ${ }^{22}$. In addition, past EEG and fMRI studies investigated age-related changes in cortical electrical activity and cerebral blood flow and showed a decrease in power during aging with both linear and nonlinear trends ${ }^{23,24}$. Taking into account both past and present findings, we propose a hypothesis that accumulated impairment of cortical electrical activity first manifests as an increase in the NPV value prior to phase transition from the healthy state to the disease state and then as a decrease in the Power value after the phase transition to the disease state (Fig. 4).

The present study demonstrated that, unlike other neuroimaging methods such as SPECT, PET, fMRI, and traditional EEG power analysis and eLORETA-power analysis that have been used to explore brain activity, eLORETA-NPV analysis had the capacity to sensitively detect changes in fluctuations of cortical electrical activities associated with CSF shunt response at the high-convexity area in iNPH. This may be attributed to the following: (i) EEG directly detects cortical electrical activity with high temporal resolution (1-2ms); (ii) eLORETA reconstructs cortical electrical activity from EEG data with correct localization; and (iii) NPV sensitively detects instability of cortical electrical activity. Our results, together with those of previous studies, suggest that eLORETA-NPV analysis is a useful and sensitive tool to assess cortical states, providing valuable information regarding the early detection of neuropsychiatric diseases and prediction of therapeutic effect.

It should be noted that our results should be interpreted with caution since the sample size of iNPH patients was relatively small. Therefore, our findings should be considered as preliminary, and would need to be confirmed in larger samples. Nevertheless, our finding of NPV changes related to the CSF shunt response is in accordance with previous neuroimaging findings in iNPH patients ${ }^{15,19-21}$. This leads us to suggest that eLORETA-NPV with the closed-form estimator is a reliable method of detecting instability of cortical electrical activity.

Overall findings indicate that NPV is an independent dimension orthogonal to 
Power and it will also contain a great deal of information about cortical electrical activity. While the classical moment estimator of NPV was problematic given the slow convergence to the true value, this issue was significantly improved by the closed-form estimator of NPV. Furthermore, eLORETA-NPV has the advantage of being a sensitive early warning signal of cortical impairment. Therefore, eLORETA-NPV analysis with the closed-form estimator is a powerful and promising tool to assess and predict brain cortical states in patients with various neuropsychiatric diseases.

\section{Methods}

\section{Subjects}

Right-handed definite and probable iNPH patients were consecutively recruited from the Neuropsychology Clinic at the Department of Psychiatry in Osaka University Hospital. The iNPH patients underwent a CSF shunt operation at the Department of Neurosurgery in Osaka University Hospital between April 2010 and February 2021. The inclusion and exclusion criteria for definite and probable iNPH patients complied with Japanese diagnostic criteria for iNPH (further details can be found in our previous study $)^{15}$. All of the included patients showed the morphological feature of a disproportionately enlarged subarachnoid-space hydrocephalus on head MRI findings, as defined in Japanese diagnostic criteria for $\mathrm{iNPH}^{16}$. CSF tap test and the CSF shunt operation were performed in accordance with the Japanese Clinical Guidelines for $\mathrm{iNPH}^{16}$.

Out of 47 patients who met the inclusion criteria, three patients were excluded due to comorbidity of Parkinson's disease or parkinsonian syndrome; two patients due to long-standing overt ventriculomegaly on head MRI findings; five patients due to the lack of 500-second (500-s) artifact-free segments; and one patient that did not complete the follow-up after the CSF shunt operation. As a result, a total of 36 patients were included in the present study.

This study was approved by the Ethics Committee of Osaka University Hospital and written informed consent was obtained from patients or their families.

\section{Assessment of Gait and Cognition}

Assessments of gait and cognition have already been described in our previous study ${ }^{17}$. Briefly, gait disturbance was assessed by the Gait Status Scale-Revised

$267(\mathrm{GSSR})^{25}$, the 10-metre reciprocating walking test (WT), and the 3-metre Timed Up and 268 Go (TUG) test ${ }^{26}$. The thresholds of improvement were set at 1-point improvement in the 269 GSSR and $10 \%$ improvement in the WT and TUG. Improvement in all gait tests was 
considered clinical improvement in gait.

Cognitive impairment was assessed using the Frontal Assessment Battery (FAB) ${ }^{27}$, Mini-Mental State Examination (MMSE) ${ }^{28}$, Wechsler Adult Intelligence Scale-III (WAIS-III)-Block Design, WAIS-III-Digit Symbol Coding ${ }^{29}$, Wechsler Memory ScaleRevised (WMS-R)-Attention/Concentration Index ${ }^{30}$, and Trail Making Test Part A (TMT-A) ${ }^{31}$. The improvement thresholds of the cognitive tests were set at 2, 3, 3, 3, 15 points, and $30 \%$, respectively. Improvement in more than half of the cognitive tests was considered clinical improvement in cognition.

278

\section{Assessment of CSF shunt operation outcome}

The method of assessment of the outcome of the CSF shunt operation has already been described in our previous study ${ }^{17}$. Briefly, gait and cognitive symptoms were evaluated 1-3 months before the CSF shunt operation - and again 1, 3, 6 months and 1 year after the operation. CSF shunt operation outcome was deemed positive if gait or cognition had clinical improvement at any time of postsurgical evaluations; otherwise, it was deemed negative. Accordingly, the patients were classified into responders and nonresponders to the CSF shunt operation. These classification criteria were stricter than those of the Japanese Clinical Guidelines for $\mathrm{iNPH}^{16}$ as we only selected those patients that had shown a significant improvement in symptoms as shunt responders ${ }^{17}$.

\section{EEG recording}

292

The EEG data acquisition, eLORETA and eLORETA-NPV analysis procedures have already been described in detail in our previous studies ${ }^{4,15}$. Briefly, EEG data was recorded during resting state with eyes closed for about 20 minutes 1-3 months before the CSF shunt operation, using a 19-electrode EEG system (EEG-1000/EEG-1200, Nihon Kohden Inc., Tokyo, Japan) with a sampling rate of $500 \mathrm{~Hz}$, and filtered through a band-pass filter of $0.53-120 \mathrm{~Hz}$. The subjects were instructed to relax in the bed but stay awake. During the EEG recordings, drowsiness was avoided by repeating the instructions. Then, 500-s artifact-free segments were selected offline by visual inspection for each patient, the band-pass filter setting was changed to $1.6-60 \mathrm{~Hz}$, and the segments were imported into the eLORETA and eLORETA-NPV analyses.

\section{eLORETA and ELORETA-NPV analyses}

eLORETA is a linear weighted minimum norm inverse solution which has the property of correct localization albeit with low spatial resolution ${ }^{2,3}$. The solution space 
realistic head model ${ }^{32}$ with the MNI152 template ${ }^{33}$. The eLORETA method has been widely used to investigate cortical electrical activities and it has been validated through studies of neuropsychiatric diseases and human data during various sensory stimulations $s^{4-6,34,35}$. In the present study, eLORETA and eLORETA-NPV analyses were performed in MATLAB R2021a software. In these analyses, for each subject, 500-s EEG data from 19 electrodes were re-referenced to the average reference to reduce diffuse artifacts covering all electrodes. EEG data were transformed into frequency data as electrode position $\times$ time $\times$ frequency matrix by discrete Fourier transform with rectangular window using time-frequency analysis ( $\mathrm{ft}$ ffreqanalysis) in Field Trip toolbox (http://www.fieldtriptoolbox.org/) installed in the MATLAB software. The frequency data were then transformed to the cortical electrical current density data as cerebral cortex position $\times$ time $\times$ frequency matrix by multiplying the spatial filter of eLORETA. The spatial filter of eLORETA was calculated using the MATLAB program written by author R. B.., which followed a technical instruction about eLORETA provided by author R. D. P.-M. ${ }^{3}$. Finally, the cortical electrical activity was obtained by squaring the cortical electrical current density and averaging over the entire EEG segment. In eLORETA-NPV analysis, NPV of cortical electrical activity for each $4.6 \mathrm{~s}$ EEG segment was calculated and averaged over the entire EEG segment, where NPV was defined as variance of cortical electrical activity divided by the square of mean cortical electrical activity. The eLORETA and eLORETA-NPV analyses were computed for five frequency bands: delta $(3.0-4.0 \mathrm{~Hz})$, theta $(4.5-7.0 \mathrm{~Hz})$, alpha $(7.5-13.0 \mathrm{~Hz})$, beta $(13.5-29.5 \mathrm{~Hz})$, and gamma $(30.0-40.0 \mathrm{~Hz})$.

\section{Information geometry about cortical electrical activity}

The cortical electrical activity at each cortical voxel in each frequency band approximately follows gamma distribution ${ }^{9}$.

$$
f(x)=\frac{1}{\Gamma(\kappa) \theta^{\kappa}} x^{\kappa-1} e^{-\frac{x}{\theta}} \quad \text { for } x>0 \quad \kappa, \theta>0
$$

where $\Gamma$ is a gamma function.

This gamma distribution is a probability distribution parameterized by a shape parameter $\kappa$ and a scale parameter $\theta$. Interestingly, this shape parameter $\kappa$ corresponds to $1 / \mathrm{NPV}$. Information geometry defines "Divergence", which is a weaker notion of distance between probability distributions, as similar distributions have small divergence and different distributions have large divergence. According to the Divergence, probability distributions are assigned to certain coordinates on the Riemannian manifold, whose geometry is characterized by a metric tensor. The metric 
tensor, which can be calculated from the second derivative of log-normalizer, induces an inner product space that allows us to measure lengths and angles between tangent vectors. In our study, we selected mixed parametrization of natural and dual parametrization as $\left(\kappa=\frac{1}{\mathrm{NPV}}, E(x)=\kappa \theta\right)$ where $E(x)$ means expected value of power.

344 The metric tensor $\mathrm{g}$ of gamma distribution for this mixed parametrization is as shown 345 below.

$$
g=\left[\begin{array}{cc}
\psi_{1}(\kappa)-\frac{1}{\kappa} & 0 \\
0 & \frac{1}{\kappa \theta^{2}}
\end{array}\right]
$$

346 where $\psi_{1}$ is a trigamma function ${ }^{10,11}$.

347 Then, we can see that the inner product between the 1/NPV parameter and the Power 348 parameter is zero, which indicates that mixed parametrization is orthogonal. This also 349 indicates that NPV itself and Power can be orthogonal on the Riemannian manifold. 350 However, with this parametrization (NPV, Power), it is difficult to calculate Divergence manually, so we took the parametrization of (1/NPV, Power) for the Divergence calculation. Symmetrized Bregman Divergence between distribution $\mathrm{p}$ and $\mathrm{q}$ can be calculated from the metric tensor $\mathrm{g}$ as follows:

$$
\text { Divergence }=\left(\oint_{\boldsymbol{x}_{q}}^{x_{p}} d x g\right) \cdot\left(x_{p}-x_{q}\right)
$$

where $\boldsymbol{x}_{p}, \boldsymbol{x}_{q}$ are coordinates of distribution $\mathrm{p}$ and $\mathrm{q}$ on the Riemannian manifold ${ }^{10}$. Thus, symmetrized Bregman Divergence between $\left(\kappa_{1}, \kappa_{1} \theta_{1}\right)$ and $\left(\kappa_{2}, \kappa_{1} \theta_{1}\right)$ was 356 calculated as:

$$
\text { Divergence }\left(\kappa_{1}, \kappa_{1} \theta_{1} \leftrightarrow \kappa_{2}, \kappa_{1} \theta_{1}\right)=\left[\psi\left(\kappa_{2}\right)-\psi\left(\kappa_{1}\right)+\log \frac{\kappa_{1}}{\kappa_{2}}\right]\left(\kappa_{2}-\kappa_{1}\right)
$$

357 where $\psi$ is a digamma function.

358 Symmetrized Bregman Divergence between $\left(\kappa_{2}, \kappa_{1} \theta_{1}\right)$ and $\left(\kappa_{2}, \kappa_{2} \theta_{2}\right)$ was calculated as:

$$
\text { Divergence }\left(\kappa_{2}, \kappa_{1} \theta_{1} \leftrightarrow \kappa_{2}, \kappa_{2} \theta_{2}\right)=\kappa_{2}\left(\frac{1}{\kappa_{1} \theta_{1}}-\frac{1}{\kappa_{2} \theta_{2}}\right)\left(\kappa_{2} \theta_{2}-\kappa_{1} \theta_{1}\right)
$$
calculated as shown below (since the Pythagorean Theorem holds for Divergence):

$$
\begin{aligned}
\text { Divergence } & \left(\kappa_{1}, \kappa_{1} \theta_{1} \leftrightarrow \kappa_{2}, \kappa_{2} \theta_{2}\right) \\
& =\text { Divergence }\left(\kappa_{1}, \kappa_{1} \theta_{1} \leftrightarrow \kappa_{2}, \kappa_{1} \theta_{1}\right) \\
& + \text { Divergence }\left(\kappa_{2}, \kappa_{1} \theta_{1} \leftrightarrow \kappa_{2}, \kappa_{2} \theta_{2}\right)
\end{aligned}
$$




\section{Closed-form estimator of NPV and $\boldsymbol{\theta}$}

We adopted a closed-form estimator of NPV and $\theta$, which were obtained by applying maximum likelihood estimation to a generalized gamma distribution. The great advantage of this is that it allows a significantly faster convergence to the true value than with a classical moment estimator, which we used in a previous study ${ }^{12,15}$.

367 We also adopted bias correction to obtain unbiased estimators of NPV and $\theta$, as 368 follows $^{12}$ :

$$
\frac{n}{n-1} E(\widehat{N P V})=N P V, \quad \frac{n}{n-1} E(\widehat{\theta})=\theta
$$

369

370

371

372

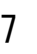

where $\mathrm{n}$ is a number to be averaged, $\widehat{N P V}$ and $\widehat{\theta}$ are closed-form estimators, and NPV and $\theta$ are their true values.

\section{Statistical group analysis}

Before statistical comparison, the eLORETA cortical electrical activity of each subject was normalized by dividing by its mean value over whole cortical voxels and frequency bands to lessen subject-wise differences in cortical electrical activity (subjectwise normalization). We measured the statistical group difference of the eLORETA cortical electrical activity between shunt responders and non-responders at each cortical voxel in each frequency band using G-test with the symmetrized Bregman Divergence calculated above $\mathrm{e}^{36}$. The level of significance for the G-test was set at $p<0.05$ with multiple comparison correction across 6239 cortical voxels using a permutation test ${ }^{37}$. Gamma distribution of eLORETA cortical electrical activity of each frequency band followed different shape and scale parameters; therefore, we applied the permutation test to the 6239 cortical voxels but not to the five frequency bands.

\section{Data availability}

Data are available from the corresponding author upon reasonable request. 


\section{References}

1. Gloor, P. Hans Berger and the discovery of the electroencephalogram. Electroencephalogr. Clin. Neurophysiol. 28, 1-36 (1969).

2. Grech, R., C. et al. Review on solving the inverse problem in EEG source analysis. J. Neuroeng. Rehabil. doi: 10.1186/1743-0003-5-25 (2008).

3. Pascual-Marqui, R.D. Discrete, 3D distributed, linear imaging methods of electric neuronal activity. Part 1: exact, zero error localization. arXiv :0710.3341 [math-ph], 2007October-17, http://arxiv.org/pdf/0710.3341 (2007).

4. Aoki, Y. et al. Detection of EEG-resting state independent networks by eLORETAICA method. Front. Hum. Neurosci. doi: 10.3389/fnhum.2015.00031 (2015).

5. Pascual-Marqui, R.D. et al. Comparing EEG/MEG neuroimaging methods based on localization error, false positive activity, and false positive connectivity. bioRxiv. doi: https://doi.org/10.1101/269753 (2008).

6. Pascual-Marqui, R.D. et al. Assessing interactions in the brain with exact lowresolution electromagnetic tomography. Philos. Trans. A. Math. Phys. Eng. Sci. 369 (1952), 3768-3784. doi: 10.1098/rsta.2011.0081 (2011).

7. Babiloni, C. et al. Abnormalities of resting-state functional cortical connectivity in patients with dementia due to Alzheimer's and Lewy body diseases: an EEG study. Neurobiol. Aging 65, 18-40. doi: 10.1016/j.neurobiolaging.2017.12.023 (2018).

8. Canuet, L. et al. Resting-state EEG source localization and functional connectivity in schizophrenia-like psychosis of epilepsy. PLoS. One 6 (11), e27863. doi: 10.1371/journal.pone.0027863 (2011).

9. Thatcher, R.W., North, D. \& Biver, C. Evaluation and validity of a LORETA normative EEG database. Clin. EEG Neurosci. 36 (2), 116-122. doi: 10.1177/155005940503600211 (2005).

10. Amari, S. \& Nagaoka, H. Methods of information geometry. (Oxford: American Mathematical Society/Oxford University Press, 2000).

11. Nielsen, F. An Elementary Introduction to Information Geometry. Entropy (Basel) 22 (10), 1100. doi: 10.3390/e22101100 (2020).

12. Zhi-Sheng, Y.E. \& Nan, C. Closed-Form Estimators for the Gamma Distribution Derived from Likelihood Equations. The American Statistician 2, 177-181 (2017).

13. Chen, L., Liu, R., Liu, Z.P., Li, M. \& Aihara, K. Detecting early-warning signals for sudden deterioration of complex diseases by dynamical network biomarkers. Sci. Rep. 2, 342. doi: 10.1038/srep00342 (2012).

14. Aoki, Y. et al. Normalized power variance change between pre-ictal and ictal phase of an epilepsy patient using NAT analysis: a case study. Annu. Int. Conf. IEEE Eng. 
Med. Biol. Soc. 2013, 437-440. doi: 10.1109/EMBC.2013.6609530 (2013).

15. Aoki, Y. et al. Normalized power variance of eLORETA at high-convexity area predicts shunt response in idiopathic normal pressure hydrocephalus. Sci. Rep. 10 (1), 13054. doi: 10.1038/s41598-020-70035-9 (2020).

16. Mori, E. et al. Guidelines for management of idiopathic normal pressure hydrocephalus: second edition. Neurol. Med. Chir. (Tokyo). 52 (11), 775-809. doi: 10.2176/nmc.52.775 (2012).

17. Aoki, Y. et al. EEG and Neuronal Activity Topography analysis can predict effectiveness of shunt operation in idiopathic normal pressure hydrocephalus patients. Neuroimage Clin. 3, 522-530. doi: 10.1016/j.nicl.2013.10.009 (2013).

18. Musha, T. et al. EEG markers for characterizing anomalous activities of cerebral neurons in NAT (neuronal activity topography) method. IEEE Trans. Biomed. Eng. 60 (8), 2332-2338. doi: 10.1109/TBME.2013.2255101 (2013).

19. Narita, W. et al. High-Convexity Tightness Predicts the Shunt Response in Idiopathic Normal Pressure Hydrocephalus. AJNR Am. J. Neuroradiol. 37 (10), 1831-1837. doi: 10.3174/ajnr.A4838 (2016).

20. Virhammar, J., Laurell, K., Cesarini, K.G. \& Larsson, E.M. The callosal angle measured on MRI as a predictor of outcome in idiopathic normal-pressure hydrocephalus. J. Neurosurg. 120 (1), 178-184. doi: 10.3171/2013.8.JNS13575 (2014).

21. Virhammar, J., Laurell, K., Cesarini, K.G. \& Larsson, E.M. Preoperative prognostic value of MRI findings in 108 patients with idiopathic normal pressure hydrocephalus. AJNR Am. J. Neuroradiol. 35 (12), 2311-2318. doi: 10.3174/ajnr.A4046 (2014).

22. Ishii, R. et al. Healthy and Pathological Brain Aging: From the Perspective of Oscillations, Functional Connectivity, and Signal Complexity. Neuropsychobiology 75 (4), 151-161. doi: 10.1159/000486870 (2017).

23. Allen, E.A. et al. A baseline for the multivariate comparison of resting-state networks. Front. Syst. Neurosci. 4; 5: 2. doi: 10.3389/fnsys.2011.00002 (2011).

24. Babiloni, C. et al. Sources of cortical rhythms in adults during physiological aging: a multicentric EEG study. Hum. Brain Mapp. 27 (2), 162-172. doi: 10.1002/hbm.20175 (2006).

25. Kubo, Y. et al. Validation of grading scale for evaluating symptoms of idiopathic normal-pressure hydrocephalus. Dement. Geriatr. Cogn. Disord. 25 (1), 37-45. doi: 10.1159/000111149 (2008).

26. Podsiadlo, D. \& Richardson, S. The timed "Up \& Go": a test of basic functional 
mobility for frail elderly persons. J. Am. Geriatr. Soc. 39 (2), 142-148. doi: 10.1111/j.1532-5415.1991.tb01616.x (1991).

27. Dubois, B., Slachevsky, A., Litvan, I. \& Pillon, B. The FAB: a Frontal Assessment Battery at bedside. Neurology 55 (11), 1621-1626. doi: 10.1212/wnl.55.11.1621 (2000).

28. Folstein, M.F., Folstein, S.E. \& McHugh, P.R. "Mini-mental state". A practical method for grading the cognitive state of patients for the clinician. J. Psychiatr. Res. 12 (3), 189-198. doi: 10.1016/0022-3956(75)90026-6 (1975).

29. Wechsler, D. Wechsler Adult Intelligence Scale-Third Edition. (San Antonio: The Psychological Corporation, 1997).

30. Wechsler, D. Wechsler Memory Scale-Revised. (San Antonio: The Psychological Corporation, 1987).

31. Reitan, R.M. The relation of the trail making test to organic brain damage. $J$. Consult. Psychol. 19 (5), 393-394. doi: 10.1037/h0044509 (1955).

32. Fuchs, M., Kastner, J., Wagner, M., Hawes, S. \& Ebersole, J.S. A standardized boundary element method volume conductor model. Clin. Neurophysiol. 113 (5), 702-712. doi: 10.1016/s1388-2457(02)00030-5 (2002).

33. Mazziotta, J. et al. A probabilistic atlas and reference system for the human brain: International Consortium for Brain Mapping (ICBM). Philos. Trans. R. Soc. Lond. B. Biol. Sci. 356 (1412), 1293-1322. doi: 10.1098/rstb.2001.0915 (2001).

34. Jatoi, M.A., Kamel, N., Malik, A.S. \& Faye, I. EEG based brain source localization comparison of sLORETA and eLORETA. Australas. Phys. Eng. Sci. Med. 37 (4), 713-721. doi: 10.1007/s13246-014-0308-3 (2014).

35. Zhang, J. et al. Switching Tinnitus-On: Maps and source localization of spontaneous EEG. Clin. Neurophysiol. 132 (2), 345-357. doi: 10.1016/j.clinph.2020.10.023 (2021).

36. McDonald, J.H. "G-test of goodness-of-fit". Handbook of Biological Statistics (Third ed.). 53-58 (Baltimore, Maryland: Sparky House Publishing, 2014).

37. Nichols, T.E. \& Holmes, A.P. Nonparametric permutation tests for functional neuroimaging: a primer with examples. Hum. Brain Mapp. 15 (1), 1-25. doi: 10.1002/hbm.1058 (2002). 


\section{Acknowledgments}

494 This study was supported by the following funding from the Research Committee of

495 Normal Pressure Hydrocephalus and Related Disorders: (i) grants for studies focusing

496 on etiology, pathogenesis and therapy from the Japanese Ministry of Health, Labour and

497 Welfare (Tokyo, Japan) (08069915, 11103508, 14428046) and (ii) a grant for studies

498 into the creation of Japanese Clinical Guidelines for iNPH from the Japanese Ministry

499 of Health, Labour and Welfare (Tokyo, Japan) (17933261).

500 This study was supported by the following grants from the Japan Agency for Medical

501 Research and Development: (i) 'Study into the possibility of hydrocephalus treatment

502 by elimination of amyloid- $\beta$ in the brain (16K10212)'; (ii) 'Study into the prediction of

503 the effect of surgery in patients with idiopathic normal pressure hydrocephalus

504 (24591710)'; (iii) 'Development and validation of artificial intelligence to detect and

505 classify prodromal dementia using EEG in resting state (JP20de0107001)'; and (iv)

506 'Study of optimization of epilepsy treatment using artificial intelligence (Brain/MINDS

507 Beyond; JP23m0307103)'.

508 This study was also supported by the following grant from Sugita Memorial Brain

509 Research Grant Funding: 'Study into gait disturbance in patients with idiopathic normal

510 pressure hydrocephalus'.

511 We are grateful to Prof. Haruhiko Kishima and staff members of the Department of

512 Neurosurgery in Osaka University Hospital, who performed CSF shunt operations.

513

514 Author contributions

515 Y.A., Hiroaki K., and R.I. designed the study. Y.A. wrote an eLORETA-NPV program, 516 analysed the EEG data and wrote the manuscript. R.D.P.-M. and R.B. provided the 517 eLORETA program and gave technical advice about it and the Field Trip toolbox. H.K., 518 K.Y., T.W., H.K., Y.S., T.S., Y.S., and M.Y. recruited subjects, performed CSF tapping 519 and assessed their symptoms. All authors reviewed the manuscript.

520

521 Competing interests

522 The authors declare no competing interests. 


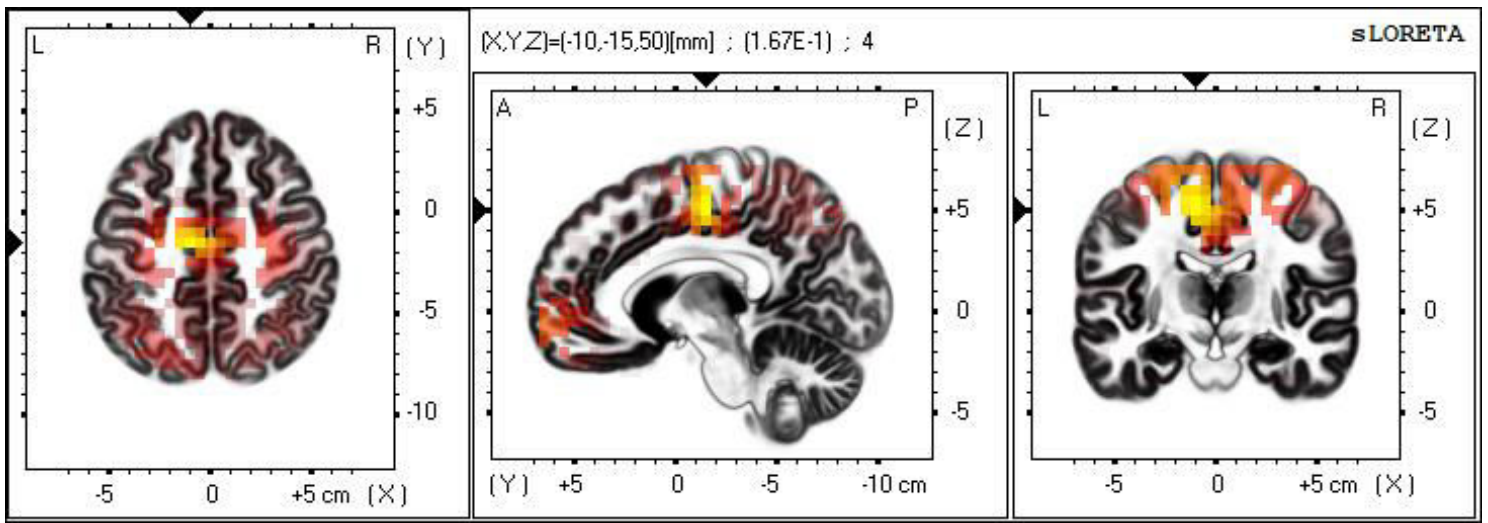

525 Figure 1. Mean eLORETA-NPV values of 17 shunt responders in the beta frequency band. Normalized power variance (NPV) of eLORETA cortical electrical activity is

527 colour coded from grey (zero) to red to bright yellow (maximum). Slices from left to right are axial, sagittal, and coronal images (viewed from top, left and back). L, left; R, right; $\mathrm{A}$, anterior; $\mathrm{P}$, posterior. 


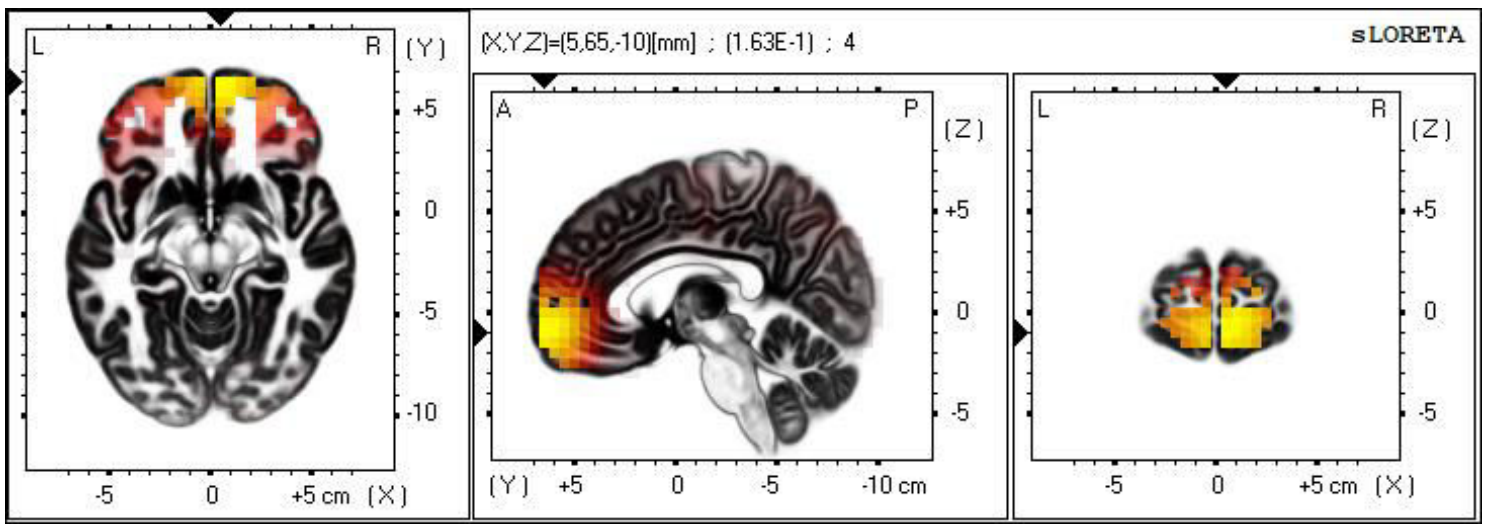

534 Figure 2. Mean eLORETA-NPV values of 19 non-responders in the beta frequency

535 band. Normalized power variance (NPV) of eLORETA cortical electrical activity is

536 colour coded from grey (zero) to red to bright yellow (maximum). Slices from left to

537 right are axial, sagittal, and coronal images (viewed from top, left and back). L, left; R, 538 right; $\mathrm{A}$, anterior; $\mathrm{P}$, posterior. 


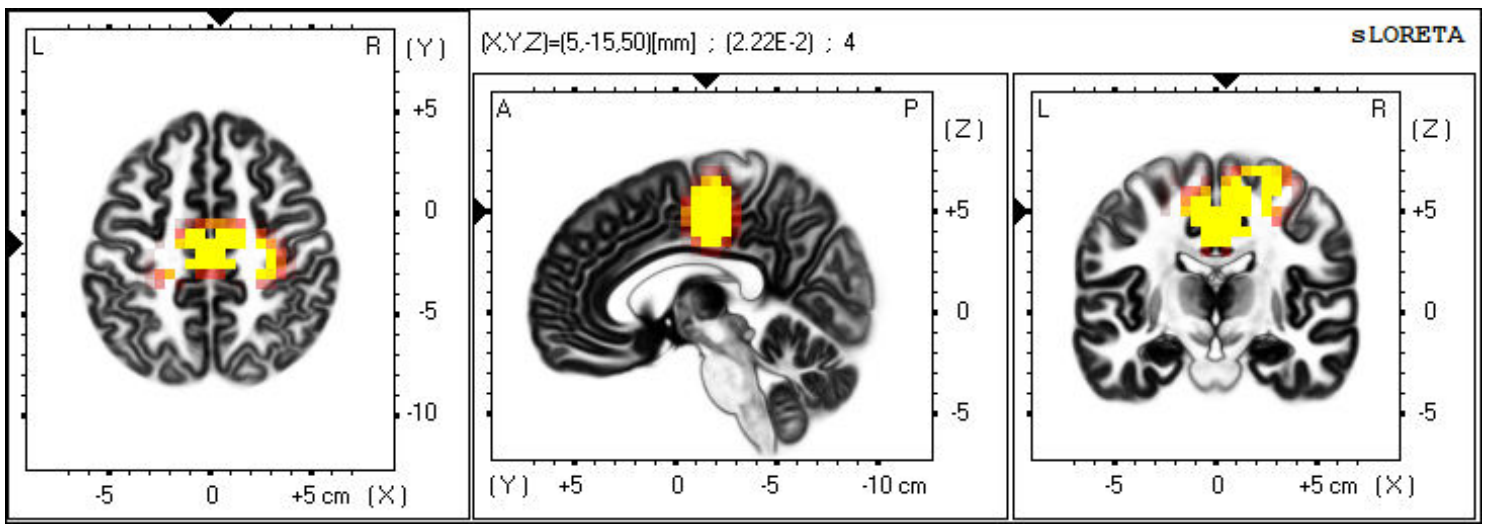

542 Figure 3. The significant difference in the normalized power variance (NPV) of

543 eLORETA cortical electrical activity-between 17 shunt responders and 19 non-

544 responders - in the beta frequency band, measured by G-test with the symmetrized

545 Bregman Divergence. Shunt responders had significantly higher NPV values of cortical

546 electrical activity in the beta frequency band at the high-convexity area compared to

547 non-responders [extreme $p=0.016$ at the paracentral lobule $(\mathrm{X}=5, \mathrm{Y}=-15, \mathrm{Z}=50$

548 (mm); MNI coordinates)]. The symmetrized Bregman Divergence above threshold ( $p<$

5490.05 ) is colour coded from red to bright yellow (maximum). Slices from left to right are

550 axial, sagittal, and coronal images (viewed from top, left and back). L, left; R, right; A,

551 anterior; $\mathrm{P}$, posterior.

552

553 


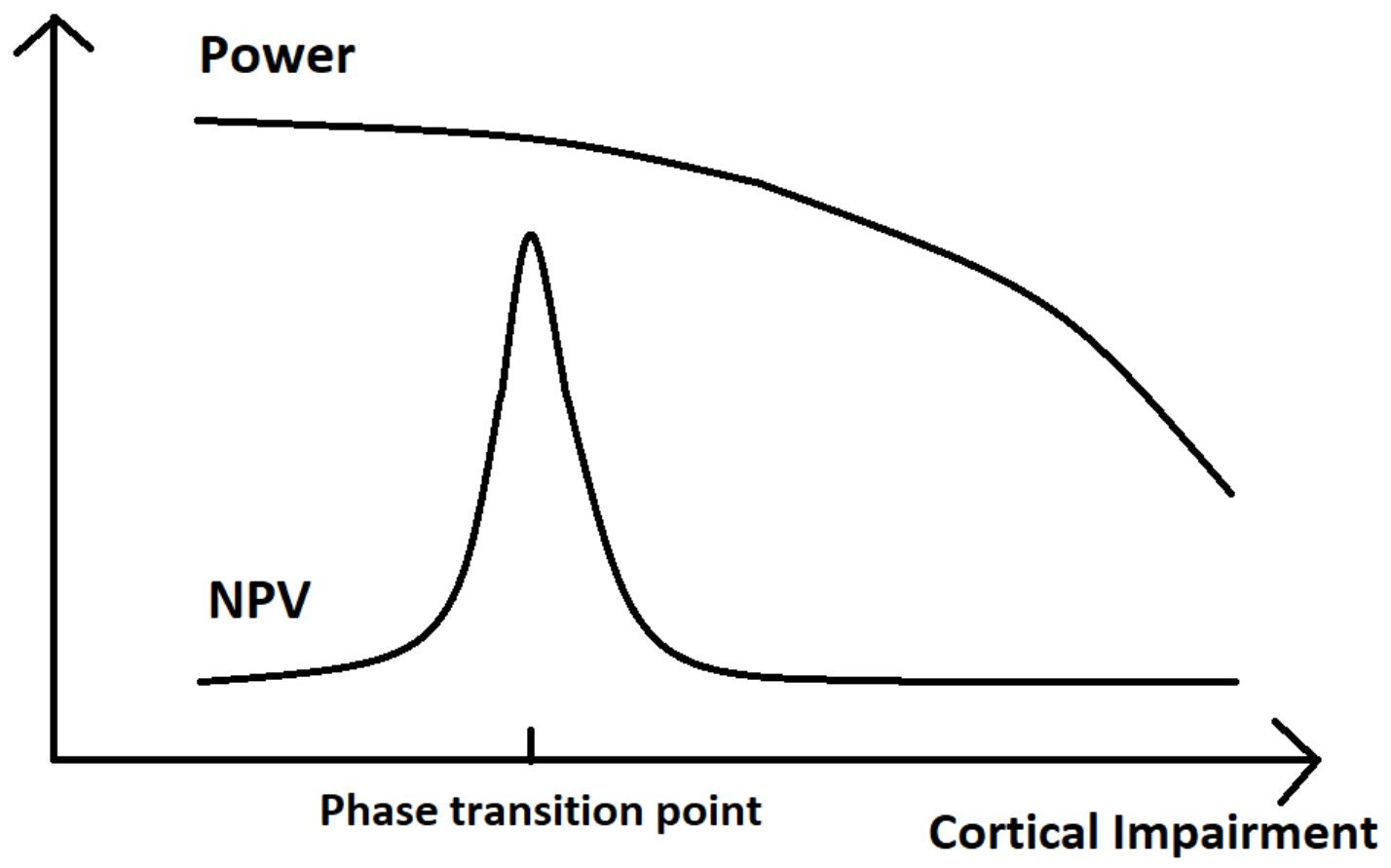

555 Figure 4. Hypothesis regarding changes in NPV and Power of cortical electrical activity 556 for accumulated cortical impairment. Impaired cortical electrical activity first manifests 557 as an increase in NPV value prior to phase transition from the healthy state to the 558 disease state and then as a decrease in Power value after the phase transition to the 559 disease state. 
Table 1.

562 Cognitive and gait function test scores of shunt responders and non-responders

\begin{tabular}{|l|c|c|c|}
\hline Test & Shunt responders & Non-responders & $p$-value \\
\hline Gender (F/M) & $7 / 10$ & $8 / 11$ & 0.95 \\
\hline Age & $76.0 \pm 6.0$ & $76.0 \pm 6.0$ & 0.75 \\
\hline WT & $26.6 \pm 18.5$ & $21.8 \pm 6.3$ & 0.36 \\
\hline TUG & $15.6 \pm 5.5$ & $13.7 \pm 4.9$ & 0.27 \\
\hline GSSR & $7.1 \pm 3.2$ & $6.0 \pm 2.0$ & 0.21 \\
\hline MMSE & $23.4 \pm 4.1$ & $24.4 \pm 3.9$ & 0.44 \\
\hline FAB & $11.2 \pm 2.6$ & $11.8 \pm 2.6$ & 0.45 \\
\hline TMT-A & $110 \pm 76$ & $105 \pm 90$ & 0.86 \\
\hline $\begin{array}{l}\text { WMS-R_Attention/ } \\
\text { Concentration index }\end{array}$ & $82.4 \pm 16.4$ & $85.7 \pm 12.1$ & 0.50 \\
\hline $\begin{array}{l}\text { WAIS-IIFDigit Symbol- } \\
\text { Coding }\end{array}$ & $6.5 \pm 2.9$ & $7.2 \pm 2.6$ & 0.51 \\
\hline WAIS-IIFBlock Design & $6.9 \pm 3.0$ & $7.3 \pm 2.1$ & 0.67 \\
\hline
\end{tabular}

563 Data are mean \pm SD. Difference in the female to male ratios between shunt responders

564 and non-responders was assessed using Chi-square test. Differences in the other scores

565 are assessed using independent Student's t test (uncorrected). WT: 10-metre 566 reciprocating walking test, TUG: $3 \mathrm{~m}$ Timed Up and Go, GSSR: Gait Status Scale567 Revised, MMSE: Mini-Mental State Examination, FAB: Frontal Assessment Battery, 568 TMT-A: Trail Making Test Part A, WMS-R: Wechsler Memory Scale-Revised, WAIS569 III: Wechsler Adult Intelligence Scale-III. 